American Journal of Pharmaceutical Education 2017; 81 (1) Article 1.

\title{
VIEWPOINTS
}

\section{Reflections for a Year of Caring and Understanding}

\author{
Gayle A. Brazeau, $\mathrm{PhD}^{\mathrm{a}, \mathrm{b}}$ \\ ${ }^{a}$ Editor, American Journal of Pharmaceutical Education, Alexandria, Virginia \\ ${ }^{\mathrm{b}}$ University of New England, College of Pharmacy, Portland, Maine
}

As we start 2017 after a most interesting 2016, I hope you will grant me the liberty to take a few minutes of your time to read a viewpoint that is philosophical in nature. As faculty members and/or administrators, we continue to solve existing and new challenges as we strive for excellence in the education of our undergraduate students, doctor of pharmacy students, graduate students, residents, and post-doctoral fellows. We also continue to work to promote the success of our fellow academicians and their work in advancing the biomedical, pharmaceutical, clinical, and social administrative sciences necessary for promoting and enhancing pharmacy practice. Perhaps you have a different perspective, but I find it discouraging watching the increasing rhetoric and too many events demonstrating the lack of or unwillingness to value what each individual brings to his or her community. I reflect in these situations on what small things we can do as educators to demonstrate to those in our colleges and schools of pharmacy that we value each individual for their unique backgrounds, perspectives, beliefs, and talents and truly care for their individual successes and the collective successes of our institution. I respectfully propose we can make small changes by refocusing our thinking, thus providing the foundation for enhancing our understanding and caring for others.

Understanding and care require deep listening, which can only occur if we are really present in our interactions with others. I would encourage us to have more personal interactions with our colleagues that don't involve computers or mobile devices, whether it is for one-on-one interactions, meetings or seminars. If we truly focus on listening to one another and what is happening in a meeting or seminar rather than a new e-mail or other work, then we will be able to better observe the non-verbal cues of others around us. Turning off or not bringing a personal electronic device is unlikely to change the course of any given day or project. Furthermore, all of us have experienced what we perceived to be a lack of caring or understanding when talking with another when they are focused more on their own phone or other mobile device. What potential new relationships could we develop or what new knowledge or perspective could we acquire if we were just to be in the present with those around us? I challenge each of you to start by having just one meeting per week where phones and other electronic devices are not allowed. Use that time to focus on what you can learn or better understand.

Understanding and caring require us to think about our words and to move by leaving our desk and talking with others outside our offices, classrooms, laboratories, and clinics. Words and how they are spoken matter particularly in a time of stress and anxiety in our colleges and schools of pharmacy. We all can remember a time when something spoken caused us to become disengaged and discouraged. The other individual may not have even known the impact of these words, but these can have long-lasting repercussions in our relationships with others.

Yet, perhaps these instances can be minimized if we take the time to get to know our colleagues outside of any e-mail trails, meetings or teaching activities. How many of us take the time to go for coffee or to lunch with our colleagues? I would think that most of us would agree that the most important relationships that we have developed have resulted by spending time together when we can share and learn from each other in an open and positive environment. I have always found it compelling during a memorial service when faculty colleagues share touching stories about a colleague who recently passed and the time they spent together at lunch, in a faculty lounge or other settings outside their offices and classrooms. They rarely reminisce about the times together while in meetings or in classrooms. What has caused us to be too busy to find time for a cup of coffee or lunch with a colleague?

Understanding and caring require us to read broadly and to challenge pre-existing assumptions. We emphasize in our students the importance of professional reading, be it textbooks, clinical protocols or evidence-based literature as the foundation for a successful practice. Yet, to what extent do we take the time to read within and outside our primary areas of clinical, scientific, and educational expertise? Reading can provide the background for rethinking our assumptions. I have found that reading broadly in professional literature and across other genres 


\section{American Journal of Pharmaceutical Education 2017; 81 (1) Article 1.}

expands my knowledge and helps me expand my perspectives. I can consider new perspectives and revise my existing assumptions when I am exposed to other points of view. I would ask if your assumptions or the collective assumptions of a group are still valid in your present situation. Challenging situations may require us to take a careful look at all assumptions, particularly assumptions that blind our abilities to look for new solutions. Our assumptions, either conscious or unconscious, form the foundation of our interactions with others and how we approach solutions involving others. We, as educators, need to demonstrate to our students and to our faculty colleagues that we value the diversity, beliefs, and talents of those with whom we travel in this educational adventure. It is our differences in lived experiences that can bring one or more perspectives to form the assumptions used in any decision-making process.

Understanding and caring require us to be willing to ask what we are willing to say "goodbye" to and what are we willing to say "hello" to. We all have processes of how things are to be done in systems that are optimized for achievement of our goals and projects. Each of us has biases, and we easily remember those situations when our interactions with others were less than positive. It is easy to assume that the only way to complete a project or task is the way we have done it in the past. Furthermore, based on our previous interactions with others that were not positive, we are less than agreeable or open to work with these individuals in new situations. We certainly need to learn from past experiences, but can we limit ourselves and our organizations if we are not willing to consider how to say goodbye to how we have done it previously, and hello to something new as we approach a challenging situation?
We all have had to say goodbye to something and hello to something else in our personal lives, which in most situations results in a positive step forward.

Understanding and caring require us to take care of ourselves and to take the time to be thankful for what we have with an emphasis on all the relationships in our life. Taking time to reflect on those who have enabled us to be successful in the past and those who have enabled us to be successful in our present environment provide a time for pause and gratitude. Taking time to think about our lifestyle and what an academic career enables us to do is also a time for pause and gratitude. Have you recently taken a few minutes to stroll around your campus and be thankful for what you see during this time away from the office? We are particularly fortunate working in an academic environment where we are provided with the opportunity to learn and grow from our professional colleagues and students.

I truly believe those of us who select an academic career have a desire for being an individual who wants to be caring and understanding of others as one of our core values. We will all face challenges, often daily, in our various roles as pharmacy educators and administrators centered on interactions with our students, faculty colleagues, staff colleagues, members of our extended academic, clinical or professional environments, and our patients. These challenges are the real opportunities we have as an individual to enhance our ability for caring and understanding of those around us. As you finish reading this viewpoint, it is my hope that these simple ideas will help you to go forward with a new perspective and tools to be the caring and understanding individual you see in the mirror each morning. 